\title{
Fazit und Ausblick
}

Mit dieser Forschungsarbeit wurde eine aufdeckende Sicht eingenommen bezogen auf die zentrale Fragestellung, inwieweit sich für Social Entrepreneure in Deutschland Kriterien identifizieren und als allgemeingültige Systematik darstellen lassen, die ihre Arbeitsgestaltung in spezifischer Weise beeinflussen und dabei eventuell Hinweise auf die Wirkung von Arbeit geben. Dabei konnte ein breites Spektrum an Sinnkonstruktionen von Social Entrepreneuren in Deutschland aufgezeigt werden: von motivationalen Einstellungen über subjektive Theorien zum Unternehmerischen und zum Sozialen bis hin zu gesellschaftspolitischen Deutungsmustern. Ebenso wurde deutlich, dass eine breit angelegte sozialunternehmerische Kulturlandschaft mit jeweils eigenen Prozessdynamiken und zu bewältigenden Herausforderungen besteht: dies sowohl im Vergleich zwischen Social Entrepreneuren als auch zwischen Sozialunternehmen sowie zwischen Netzwerkstrukturen.

Das Fazit und der Ausblick zur vorgelegten Untersuchung erfolgt dabei in dreierlei Hinsicht: mit einem wissenschaftstheoretischen Fokus, mit einem methodologischen Fokus und mit einem praxisbezogenen Fokus, der (Handlungs-) Empfehlungen beinhaltet. Der damit verbundene gesellschaftliche Nutzen sowie der weitere Forschungsbedarf werden spezifiziert.

Herausgearbeitet wurde sowohl vor Erhebungsbeginn (Kap. 2) als auch in erweiterter Betrachtung mit internationalem Bezug während der Ergebnisdiskussion (ab Kap. 4), dass in verschiedenen Forschungsdisziplinen Interesse besteht, arbeits- und organisationswissenschaftlichen Erkenntnisgewinn zu verschiedenen Fragestellungen im Hinblick auf Social Entrepreneurship zu schaffen und zu erweitern. Dabei nimmt diese Dissertation mit ihrem arbeitswissenschaftlichen Schwerpunkt eine bislang weitestgehend unerforscht gebliebene Perspektive ein. Mit diesem Blickwechsel ließ sich der Erkenntnisgewinn insbesondere im Hinblick auf den Bedeutungsgehalt eines Belastungsgefüges für Arbeitskontexte von 
Sozialunternehmern erweitern. Typische Belastungssituationen, der Umgang mit sowie die Auswirkungen von Belastungssituationen konnten gezielt erforscht werden.

Das Spiegeln internationaler Forschungserkenntnisse an den eigenen Erkenntnissen zeigt, dass mehrere der in dieser Studie aufgedeckten Phänomene eine allgemeingültige Relevanz besitzen, sozusagen grenzenlos von Forschungsinteresse sind. Im internationalen Fokus steht beispielsweise die Erforschung der Persönlichkeitstypologie des Sozialunternehmers, wobei die Erforschung zur Social-Entrepreneurship-Intention bei zumeist jungen Befragten ein Schwerpunkt ist, was zu entsprechenden Limitationen führt, die in dieser Arbeit herausgearbeitet wurden. In der Gesamtbetrachtung lassen sich im internationalen Forschungsvergleich keine Erkenntnisse identifizieren, die zur Beantwortung der zentralen Leitfrage in dieser Untersuchung unberücksichtigt geblieben sind bzw. einen zusätzlichen Beitrag zur Beantwortung der zentralen Leitfrage hätten liefern können.

Ebenso bestehen Phänomene, die im Speziellen auf Deutschland zutreffen. Beispielsweise bringen die historisch gewachsene deutsche Wohlfahrtskultur und gesetzliche Vorgaben Eigenarten zu Förderbedingungen mit sich.

Darüber hinaus gibt es Phänomene, die zwar ebenfalls im internationalen Gefüge eine Rolle spielen, in Deutschland jedoch in besonderer Form in den Mittelpunkt gestellt worden sind. Dadurch haben Prägungen stattgefunden und finden weiterhin statt, die bundesweit für eine sozialunternehmerische Kulturentwicklung nicht nur lenkend sind, sondern einschränkende Wirkung entfalten: Hochskalierungsansprüche, ein Innovationspostulat und vielerorts erzeugter Problemlösungsdruck sind hierzu drei Beispiele.

In der zentralen Forschungsfrage stecken Teilfragen, die herausgearbeitet und intensiv bearbeitet worden sind, um das Gesamtproblem zu verstehen. Empirisch fundiert hergeleitet und konzeptionell diskutiert wurde, aus welchen Puzzlesteinen das Gesamtbild besteht und in welchen Lagebeziehungen diese Puzzleteile zueinanderstehen (können). In der Gesamtbetrachtung zeigte sich ein vielschichtiges Bedingungsgefüge an Belastungssituationen bei der Arbeitsgestaltung und in der Wirkung von Arbeit bei Social Entrepreneuren mit wiederum typischen Merkmalen, die in der übergeordneten Struktur als ein Wirkungsmodell zur Arbeitsgestaltung von Social Entrepreneuren in Deutschland dargestellt werden konnten, wiedergegeben mit Abbildung 11.1.

Herausgearbeitet wurde dabei zum einen, dass und wie Belastungen auf das Wohlbefinden von Sozialunternehmern positiv wie negativ einwirken, und zum anderen, wie sich diese Belastungen auf ihre Sozialunternehmen positiv wie negativ auswirken können. Zum Bedeutungsgehalt negativer Auswirkungen sei 


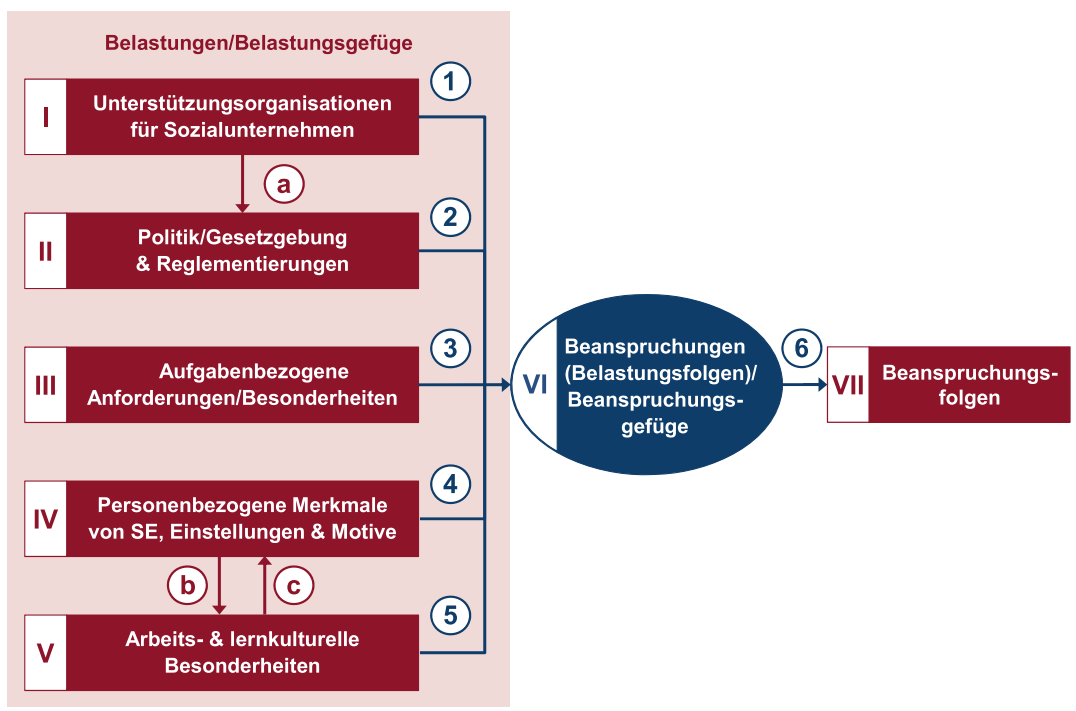

Abbildung 11.1 Wirkungsmodell zur Arbeitsgestaltung von Social Entrepreneuren als Belastungs-Beanspruchungs-Gefüge. (eigene Darstellung)

bemerkt, dass sich Interviewpartner bei diesen Schilderungen über die ohnehin vor Befragungsbeginn zugesicherte Anonymisierung hinaus vielfach nochmals ausdrücklich vergewissert haben, dass ihre entsprechende Ausführung anonymisiert wird. Darüber hinaus haben Befragte mehrfach darauf bestanden, dass bestimmte Schilderungen zu Fehlbelastungen und zu daraus hervorgegangenen Folgen nicht im direkten Kontext zu ihrem Interview veröffentlicht werden. Die betreffenden Inhalte unterstreichen weniger Einzelschicksale als vielmehr in Variation wiederkehrende und bei genauerer Betrachtung typische Fehlbelastungssituationen, so z. B. zu Mängeln in der Arbeitsweise von Unterstützungsorganisationen und Behörden oder Blockaden durch Wohlfahrtsverbände. Somit kann bereits eine eigene Aussagekraft darin stecken, wenn befragte Sozialunternehmer bestimmte, gravierende Beispiele zu Fehlbelastungen in der Arbeitsgestaltung einerseits mit besonderer Vorsicht und Einschränkung berichten ${ }^{1569}$, es ihnen andererseits offensichtlich wichtig ist, diese Erfahrungswerte - zumeist im späteren Verlauf von Interviews und darüber hinaus ergänzend im Anschluss an die offizielle Aufzeichnung - mitzuteilen, in mehreren Fällen unter Heranziehen ergänzender Dokumente und Belegquellen. 
Vergleichbar mit der Arbeit eines Ingenieurs, der einen Prototyp in den unterschiedlichsten Alltagssituationen und unter verschiedenen Umweltbedingungen testet, konnten Qualitäten zu Bedingungen der Arbeitsgestaltung für Sozialunternehmer herausgearbeitet und analysiert werden, deren Wirksamkeit sich in kontextualen Bedingungen interpretieren und verstehen lässt. Auf Basis der daraus hervorgegangenen Erkenntnisse lassen sich Diskussionen auf einer wissenschaftstheoretischen, einer methodischen und einer praxisbezogenen Ebene anstoßen und vorantreiben. Der dabei entstehende Nutzen wird nun dargestellt, wobei ebenso auf Einschränkungen bzw. Limitationen eingegangen wird.

\subsection{Wissenschaftstheoretischer Fokus}

Mit der Entwicklung des Wirkungsmodells zur Arbeitsgestaltung von Sozialunternehmern in Deutschland ist es gelungen, einen umfassenden Überblick zum gegebenen Belastungsgefüge darzustellen. Dabei hat der für diese Untersuchung gefeilte Methodenschlüssel es ermöglicht, die Schlösser der verschiedenen Erkenntnis-Tore zu den Arbeitsgestaltungsbedingungen zu öffnen und in der Folge wissenschaftlich fundiert die Vielfalt der sozialunternehmerischen Landschaft zu erkunden. In der Gesamtbetrachtung wird erstmals ein breites Verständnis zum Wirkungsspektrum der arbeitsgestaltungsrelevanten Bedingungen von Sozialunternehmern in Deutschland aufgezeigt, das zudem typische Zusammenhänge erfasst.

In diesem Kontext ließen sich zwei Typologie-Ansätze ableiten, die sowohl Widersprüchlichkeiten als auch Druckmechanismen aufzeigen, denen Social Entrepreneure ausgesetzt werden bzw. sich selbst aussetzen können. Dabei wurde ein Neuentwurf zum Verständnis von Sozialunternehmertum entwickelt, da auf Basis einer motivationspsychologischen sowie einstellungsbezogenen Argumentation begründet wird, weswegen das Soziale und das Unternehmerische nicht als zwei Dimensionen innerhalb eines Kontinuums aufzufassen sind, so wie bislang angenommen, sondern als vier gleichwertig anzusehende, nicht in direkter Verbindung stehende Kriterien.

Hierbei wurde darüber hinaus ein ganzheitlich-prozessorientierter Präventionskonzept-Ansatz zum Umgang mit Belastungen und Belastungsfolgen in die Diskussion eingebracht und ein prozessorientiertes Qualitätsbewusstsein wurde schrittweise hergeleitet, dessen Umsetzung dazu verhelfen soll, dass ein Social Entrepreneur bzw. ein Sozialunternehmen den vielfältigen Belastungen, die beim Social Entrepreneurship in typischer Form gegeben sein können, möglichst optimal begegnen und standhalten kann. 
Wenngleich im Rahmen der Datenanalyse nicht herausgearbeitet werden konnte, wie sich die Wirkung von Social Impact im Einzelnen nachweisen lässt, konnte dennoch aufgezeigt werden, dass das komplexe Bedingungsgefüge sozialer Wirksamkeit Social Entrepreneure vor eigene Herausforderungen stellt, dabei sogar Rechtfertigungsdruck produzieren kann. Hieraus wurde der unmittelbare Forschungs- und Entwicklungsbedarf im Hinblick auf ein erweitertes Verständnis zur Messung sozialer Wirksamkeit abgeleitet. Beispielsweise wird eingefordert, dass eine durchaus ausgesprochen hohe Wirkung kleiner, nicht hochskalierender Sozialunternehmen als solche anzuerkennen ist. Ebenso ist dem Fakt Rechnung zu tragen, dass Social Entrepreneure, die für ihre Erfolge vielfach ausgezeichnet worden sind und deren Sozialunternehmen wachsen, selbst sozialer Benachteiligung unterliegen können, wenn sie von Altersarmut betroffen sind, was wiederum in einem argumentativ inkongruenten Bedingungsgefüge begründet sein kann: Es bestehen beeinträchtigende Widersprüche, inwieweit Sozialunternehmer Einnahmen aus ihren sozialunternehmerischen Aktivitäten zur eigenen finanziellen Absicherung generieren dürfen - bzw. inwieweit ihre Tätigkeit als Erwerbsarbeit, die eine angemessene Entlohnung verdient, bewertet wird.

Zudem findet in dieser Untersuchung eine Würdigung des von Maslow herausgearbeiteten Stellenwerts von Bedürfnissen und Bedürfnisbefriedigung statt. Maslows Ausführungen finden bis dato - 50 Jahre nach der Veröffentlichung - kaum Beachtung zugunsten einer deutlich älteren, obsoleten Darstellung. Die konzeptionelle Ausarbeitung Maslows, beispielsweise zur Bedeutung von Transzendenz, lässt sich passgenau für die Situationsanalyse von SE in Deutschland heranziehen.

Herausgearbeitet wurde in Kapitel 2, dass mehrere wissenschaftliche Publikationen zum Teil umfangreiche Einschränkungen in ihrem Sampling aufweisen, ohne die damit verbundenen Limitationen zu benennen, obwohl wissenschaftlicher Support besteht. Dabei stellen einige Studien zum Teil in beabsichtigter Weise wirksame Instrumente zur politischen Lobbyarbeit dar, insbesondere für Social Startups. Wissenschaftliche Berater können ggf. den begründeten Anschein erwecken, sich in der Datenerhebung und -auswertung inhaltlich zu positionieren. In der Folge kommt es zu Ausgrenzungen von Social Entrepreneuren. Denn beispielsweise werden Anforderungen an Innovation, Problemlösung und Hochskalierung normativ in einem sozialunternehmerischen Grundverständnis verortet. Mit der Datenauswertung wurde deutlich, dass diese Positionierungen vom Praxisfeld verinnerlicht und adaptiert werden: nicht nur von Verbandsstrukturen und von Unterstützungsorganisationen für Social Startups, sondern ebenfalls von einzelnen Akteuren. Wissenschaftliche Publikationen nehmen auf diese Weise Einfluss darauf, inwieweit sich Social Entrepreneure dem Sozialunternehmertum selbst 
zuordnen (dürfen) oder ausgeschlossen werden, und in der Folge, ob ihnen der Zugang zu Unterstützungsorganisationen ermöglicht oder versagt wird. Insofern lassen sich die Untersuchungserkenntnisse dieser Dissertation als Aufruf an Forschende verstehen, normativ formulierte Aussagen zur Zugehörigkeit zum - und im Umkehrschluss zum Ausschluss von - Sozialunternehmertum zu überdenken und sich bewusst zu machen, welche Konsequenzen eine dadurch bedingte Segregation für das Praxisfeld haben kann.

In der Folge ist längst ein verzerrtes, falsches Bild von Sozialunternehmertum in Deutschland entstanden, das der Komplexität des sozialunternehmerischen Feldes nicht gerecht wird und Betroffene umfassend ausgrenzt. Somit sollten z. B. Untersuchungen, die sich auf die Situationsanalyse von Social Startups konzentrieren, diese Einschränkung bereits im Studientitel erkennbar werden lassen, keinesfalls aber mit ihren Ausführungen den Anschein erwecken, allgemeingültige Aussagen für das Sozialunternehmertum in Deutschland zu treffen.

Was den im Dienst der Lobbyarbeit stehenden wissenschaftlichen Support zu Studien anbelangt, wird empfohlen, Verbandsstrukturen darin anzuleiten, empirisch einwandfrei begründete Daten zu erheben und die Verbände bzw. Auftraggeber auf dieser Basis in ihrer Zielsetzung zu stärken, Sozialunternehmertum gesellschaftlich und politisch zu festigen. Auf diesem Weg kann die Vielfalt der bestehenden Ausgrenzungen identifiziert werden, was die Notwendigkeit einer Unterstützung für Sozialunternehmertum durch die Politik sogar noch stärker gewichtet, den Verbänden also von der Sache her argumentativ entgegenkommen sollte.

In der Gesamtbetrachtung können die gewonnenen Erkenntnisse als Basis für weiterführende Untersuchungen verwendet werden: von der dienstleisterspezifischen Evaluation der Beratungsangebote für Social Entrepreneure (vgl. Kap. 5) über die genaue Analyse bürokratischer Strukturen und ihrer Auswirkungen auf die Arbeitseffizienz und -effektivität von Sozialunternehmern (vgl. Kap. 6), zur Bestandsaufnahme von Wirkungsanalysen in der sozialen Arbeit (Kap. 7) oder zu den Auswirkungen von Anspruchniveaus auf die Arbeitsleistung und Arbeitszufriedenheit (vgl. Kap. 8) bis hin zur Untersuchung des Führungsverhaltens in Sozialunternehmen aus Mitarbeitersicht (vgl. Kap. 9) - um nur einige Beispiele zu bennen. Ebenso können die gewonnenen Erkenntnisse als Vorlage dienen, um die Arbeitsgestaltungsbedingungen von Social Entrepreneuren in anderen Ländern zu erforschen. 


\subsection{Methodologischer Fokus}

Qualitative und quantitative Untersuchungen werfen einen unterschiedlichen Blick auf Daten und können sich daher in der Social-Entrepreneurship-Forschung gegenseitig befruchten bzw. gewinnbringend ergänzen (Majumdar \& Reji, 2020, S. 4 ff.). Bereits die empirisch-methodische Situationsanalyse zu den vorgestellten quantitativen und qualitativen Studien in Kapitel 2 zeigt, dass dieses Potenzial bislang nicht genutzt wird. Im Vergleich beider Erhebungsrichtungen fällt auf, dass sich keine einzige der dargestellten quantitativen Untersuchungen auf Erkenntnisse aus den qualitativen Studien bezieht und für das eigene Befragungsdesign aufgreift. Umgekehrt verhält es sich nahezu identisch; die Autoren qualitativer Studien berücksichtigen, wenn überhaupt, den gegebenen Erkenntnisgewinn aus quantitativen Untersuchungen nur am Rande. Dies entspricht weder dem wissenschaftlichen Anspruch an Recherche noch dem Standard empirischer Forschung. Beispielsweise heben Kruse, Chipeta, Surujlal und Wegge (2018) in ihrer Studie, die den alleinigen Fokus auf Sozialunternehmertum in Deutschland hat, ,the equally high importance of quantitative next to qualitative research" (S. 7) hervor. „As the majority of papers in SE-research is of a theoretical nature or applies qualitative methodologies, empirical research is not only desirable (Lee et al., 2014; Short et al., 2009) but needed in order to advance SE-research and allow the testing of proposed theories and models (Hockerts, 2015)“ (ebd., S. 25). Ein vergleichbares Verständnis liegt bei Kruse, Wach, Costa und Moriano (2018, S. 6) zugrunde. Beispielsweise hätten sich die Autoren auf Leppert (2013) beziehen können, der sich nicht nur der identischen Fragestellung bezogen auf Sozialunternehmer in Deutschland widmet, sondern darüber hinaus die identischen theoretischen Ausführungen von Ajzen (1991) für seine Erhebung zugrunde legt.

Auch die Erkenntnisse dieser Untersuchung erweitern die Basis für weiterführende Untersuchungen. Verwiesen wird hierbei auf die methodenkritische Reflexion in Kapitel 3. Zudem wurden im Rahmen der Ergebnisdiskussion, wenngleich begründet und im Sinne des gegebenen Erkenntnisinteresses zweckmäßig gestaltet, mehrfach kontingente Entscheidungen getroffen. Selbstverständlich ist eine statistische Verallgemeinerung zu den herausgearbeiteten Untersuchungsergebnissen auf die Grundgesamtheit infolge der qualitativen Untersuchung nicht möglich. Dennoch steht außer Frage, dass Differenzierungen nach den vorgeschlagenen Typologie-Ansätzen vorgenommen werden können. Allerdings ist unklar geblieben, wie diese Differenzierungen erfolgen können und in welchen wissenschaftstheoretischen Kontexten sie angebracht erscheinen. In jedem Fall 
können die im Wirkungsmodell herausgearbeiteten Zusammenhänge herangezogen werden für Faktorenanalysen und für weitere quantitative Untersuchungen zur Ermittlung repräsentativer Aussagen.

Zudem können die Erkenntnisse zu den vorgelegten Typologie-Ansätzen zur Entwicklung von Testverfahren genutzt werden, die im Praxisfeld wiederum mit weiteren Testverfahren trianguliert werden können, um die spezifischen Umstände und Bedarfe von Sozialunternehmern in Deutschland zu untersuchen. Beispielsweise könnten zur Untersuchung von Arbeitszufriedenheit oder zur Stressbewältigung passende valide Testverfahren mit den Erkenntnissen aus dieser Studie trianguliert werden. Im Ergebnis könnte dies den Blick auf notwendige Unterstützungsmaßnahmen für Social Entrepreneure in der Praxis schärfen.

Wenngleich diese Studie ihren Beitrag dazu leistet, einen Teil der bestehenden Wissenslücken zu schließen, kann sie keinen Absolutheitsanspruch erheben. Selbst bei der gewissenhaft entwickelten Fallauswahl bestanden organisatorische strukturelle Zwänge, so dass sich selbst bei der bestmöglich eingehaltenen komparativen Vorgehensweise die Existenz weiterer Fälle mit weiteren Merkmalsausprägungen, die in dieser Untersuchung unentdeckt geblieben sind, nicht ausschließen lässt. Daher ist dieses Ergebnis als Zwischenergebnis zu verstehen und es ist zu hoffen, dass das qualitativ-empirische Überprüfen und Weiterentwickeln der vorgelegten Erkenntnisse fortgeführt wird.

So ließen sich im Datenmaterial begründend keine Hinweise auf bestimmte Phänomene identifizieren wie z. B. genderspezifische Merkmale. Jedoch sind im Sample dieser Untersuchung keine Gründungen von Sozialunternehmen vertreten, die nicht aus einem bereits bekannt gewordenen sozialen Missstand, also reaktiv hervorgegangen sind. Hierbei stellt sich die Frage, inwieweit es in Deutschland dennoch zu Gründungen von Sozialunternehmen kommt, bevor sich ein sozialer Missstand angekündigt hat, und inwieweit sich hierbei ggf. weitere Merkmale herausarbeiten lassen würden, die zur Beantwortung der zentralen Forschungsfrage ihren eigenen Beitrag leisten.

Wenngleich in Kapitel 5 bis Kapitel 9 Merkmale und Bedingungen zur Arbeitsgestaltung von Sozialunternehmern herausgearbeitet werden konnten (vgl. Abb. 11.1), ist davon auszugehen, dass mehrere Erkenntnisse ebenso für konventionelle Unternehmer von Nutzen sein können bzw. auch auf sie zutreffen. Kritisch anzumerken ist, dass in dieser Untersuchung keine eindeutige, trennscharfe Abgrenzung gegenüber konventionellem Unternehmertum besteht und die erhobenen Kriterien nicht an konventionellen Unternehmern gespiegelt werden konnten. 


\subsection{Praxisbezogener Fokus}

Mit Blick auf politische und bürokratische Strukturen zeigt sich, dass es dort an Wertschätzung und Verständnis für sozialunternehmerisches Engagement mangeln kann bzw. Wertschätzung widersprüchlich gestaltet wird. Es kommt sogar erheblicher Widerstand durch politische und behördliche Strukturen zum Tragen. Wichtig und ebenso dringend notwendig ist, die bestehenden Hürden und Blockaden abzubauen, und zwar auf Basis anspruchsvoller und konkreter Ziele, deren erreichbare wie realisierbare Wirksamkeit messbar und überprüfbar ist. In diesem Kontext ist politisch die herausfordernde Aufgabe zu meistern, Sozialunternehmertum rechtlich und damit unternehmerisch umfassend zu stärken. Dabei darf die Attraktivität des gesellschaftlich bedeutsamen ehrenamtlichen Engagements nicht geschwächt werden. Als absurd erweist sich jedoch z. B. die Tatsache, dass ehrenamtliche Mitarbeiter als ordentliche Vereinsmitglieder gesetzlich gewollt „Vereinsmeierei“ betreiben und über die unternehmerische Zukunft eines Sozialunternehmens mitentscheiden können, sogar das Führungszepter übernehmen und den Gründer bzw. Sozialunternehmer aushebeln können.

Wenngleich die bestehende Lobbyarbeit, die insbesondere auf Verbandsebene erfolgt, vom Ansatz her verständlich erscheint, ist zu bemängeln, dass hiervon in aller Regel nur jüngere SE-Startups mit akademischem Hintergrund in strukturstarken Regionen profitieren. Weiter oben wurde bereits das Fazit gezogen, dass es in der Folge zu Segregationseffekten gekommen ist und weiterhin kommt. Befragte zeigen mit ihren Ausführungen auf, dass Unterstützungsorganisationen keineswegs Sprecher für die Vielfalt an Sozialunternehmern sind. Vom Anspruchsdenken her breit aufgestellte Verbände sollten ihre Systemgrenzen nicht nur identifizieren, sondern Zugangsmöglichkeiten für alle Betroffenen aktiv schaffen und Integration ermöglichen, anstatt selbst soziale Benachteiligung zu provozieren. Alternativ sollte ein Verbandsname und vor allem die Selbstdarstellung zu erkennen geben, wenn ein Verband sich nur einem Teilbereich widmet wie z. B. der Social-Startup-Förderung, insbesondere, wenn über den gesetzten Arbeitsschwerpunkt hinaus keine hinreichende Unterstützungskompetenz vorhanden bzw. verfügbar sein sollte.

Das volkswirtschaftliche Potenzial kann bei Menschen, die von sozialer Benachteiligung direkt betroffen sind, sehr hoch sein, ohne dass dies nutzbar gemacht wird. Bei direkt Betroffenen kann es speziell an Zugangsmöglichkeiten zu Unterstützungsstrukturen scheitern. Verbände sollten daher darauf achten, von sozialer Benachteiligung Betroffene auf dem Weg zu ihrem Sozialunternehmertum qualitativ hochwertig zu unterstützen bzw. im Minimum sie in der Lobbyarbeit angemessen zu inkludieren, da Menschen ohne direkte Betroffenheit 
privilegiert sind, eher den Zugang zu Unterstützungsstrukturen und Netzwerken haben können und sich somit ihrer damit gegebenen besonderen Verantwortung bewusst sein sollten.

Darüber hinaus mangelt es an spezifischen Unterstützungsstrukturen beispielsweise für Nicht-Studierende bzw. Nicht-Studierte, für Akteure im ländlichen Raum und in strukturschwachen Regionen sowie in den neuen Bundesländern. Dies stellt eine Einschränkung in der gesamtgesellschaftlichen gemeinwohlorientierten Ausrichtung dar und ist somit zielsetzungsorientiert auf den Prüfstand zu stellen.

Ein spezifischer sozialer Auftrag an das schulische Bildungswesen besteht darin, Schüler aller Schultypen per Praxisbezug über Sozialunternehmertum aufzuklären und es ihnen grundsätzlich zu ermöglichen, eigene sozialunternehmerische Interessen in Projekten zu vertiefen. Der strukturell verankerte Zugang zu Social Entrepreneurship sollte daher in Lehrplänen mit entsprechendem Praxisbezug sichergestellt werden. Deutlich wird dabei, dass Lehrkräfte hinreichend geschult sein müssen und mit Unterrichtskonzepten zu versorgen sind. Curricula könnten z. B. fächerübergreifende Lernprojekte zum Sozialunternehmertum aufnehmen. Ebenfalls bestehen bis dato keine umfassenden Förderprogramme für Schulabgänger und für Absolventen einer Berufsausbildung, die sich dem Sozialunternehmertum widmen. Somit ist im Speziellen darauf zu achten, dass Zugangsbarrieren zum Sozialunternehmertum für Schüler gezielt abgebaut werden, die beispielsweise durch finanzielle Restriktionen gegeben sein können. Dabei ist ebenso zu bedenken, dass Absolventen von Ausbildungsberufen eine andere Form der Vernetzungsunterstützung benötigen können, da sie anders organisiert sein können und durch die Einbindung in das Berufsleben nicht nur anderen Belastungssituationen ausgesetzt sind, sondern ebenso andere Erfahrungswerte mitbringen. Welches sozialunternehmerische Potenzial bei Auszubildenden, Ausbildungsabsolventen und generell bei Nicht-Studierten in dem durch den Mittelstand geprägten Wirtschaftsstandort Deutschland schlummert und zum Leben erweckt werden könnte, ist gänzlich unerforscht.

Zudem fehlt es konventionellen Organisationen mit StartupUnterstützungsangeboten an spezifischer Beratungskompetenz für Sozialunternehmer: beispielsweise bei Kammern und Stiftungen. Sozialunternehmer bekunden eben nicht nur Beratungs- und Aufklärungsbedarf zu klassischen Fragestellungen, die in vergleichbarer Form konventionelle Unternehmer betreffen, sondern darüber hinaus zu spezifischen Gegebenheiten, die für die wirtschaftliche Existenz der Sozialunternehmen von grundlegender Bedeutung sein können: von Regularien zur Gemeinnützigkeit bis hin zu speziellen zu meisternden Hürden bei der gemeinwohlorientierten Zielsetzung, insbesondere, 
wenn Social Entrepreneure einen neuen, nur für sie typischen Markt erobern wollen und dafür zunächst Pionierarbeit leisten müssen.

Deutlich geworden ist, dass der Beratungsbedarf bei Social Entrepreneuren und in Sozialunternehmen in Quantität und Qualität deutlich höher ist als das bestehende Angebot, zumal zuweilen diverse Zugangskriterien erfüllt sein müssen, um eine Fachberatung in Anspruch nehmen zu können. Dabei kommt es zu Segregationseffekten, da sich die Angebote zumeist nur auf universitäre Kontexte beziehen, die u. a. eine Zugangsschwelle für Nicht-Studierende darstellen können. Ebenso werden Social Entrepreneure, die mit ihrem Sozialunternehmen bereits in der Betriebsphase angekommen sind, mit dem für sie eigenen Beratungsbedarf ausgegrenzt.

Zuweilen bemängeln befragte Social Entrepreneure die Qualität der bestehenden speziellen Beratungs- und Unterstützungsangebote für Sozialunternehmer. Dabei wurden konzeptionelle Unschärfen im Beratungswesen und Defizite in Beraterkompetenzen deutlich: falsche Ratschläge können die Existenz eines Sozialunternehmens bedrohen bzw. zum Scheitern führen. Somit mangelt es nicht nur quantitativ in der gesamten Social-Entrepreneurship-Landschaft an geeigneten Unterstützungsangeboten, sondern es fehlen Regularien zur Qualitätssicherung der Beratungstätigkeit. Die Ausführungen von Befragten zu in Anspruch genommenen Beratungsangeboten zeigen deutlich, dass gut gemeinte Unterstützung nicht per se gut gemacht bedeuten muss. In der Schlussfolgerung ergeben sich Anforderungen an eine Qualitätskontrolle der Arbeit von Unterstützungsorganisationen.

Gerade Verbandsstrukturen könnten hier Minimalanforderungen an Beraterprofile formulieren, die auf Basis der Mitgliederbedarfe ausgearbeitet werden können. Dabei sollte allerdings darauf geachtet werden, dass nicht „der Bock zum Gärtner" gemacht wird: Abzuraten ist in aller Deutlichkeit davon, dass Berater mit Beratungsdefiziten, die sich laut Schilderungen von Befragten in Unterstützungsorganisationen intensiv engagieren können, im Alleingang Anforderungen an ein Curriculum zur Grundausbildung des „Berufsbilds Fachberater für Sozialunternehmer" entwickeln. Hier kann z. B. die Zusammenarbeit mit fachlich und methodisch kompetenten Lehrstuhlinhabern an Hochschulen und mit Weiterbildungsorganisationen, die in der Qualitätssicherung von Beraterqualifizierungen hinreichend Expertisen vorweisen, hilfreich sein. Dabei können sich die kompetenten Praxisberater, die es selbstverständlich ebenfalls gibt, identifizieren lassen und sodann in den weiteren Ausarbeitungsprozess mit ihrer Expertise eingebunden werden.

Zudem erscheint es aufgrund der Vielfalt sozialunternehmerischer Aktivitäten ratsam, beraterbezogen $\mathrm{zu}$ differenzieren, in welchen Teilgebieten ein 
Praxisberater kompetent und erfahren ist, Social Entrepreneure bzw. Sozialunternehmen zielführend unterstützend zu begleiten. Darüber hinaus könnten sich Verbandsstrukturen dafür einsetzen, dass entsprechende Qualifizierungsprogramme anerkannt werden, und im Rahmen ihrer Lobbyarbeit könnten sich Verbände dafür einsetzen, dass qualifizierte Berater bei bestehenden finanziellen Förderprogrammen der Bundesregierung registriert werden können, so dass die Verbandsmitglieder sich eine qualifizierte Fachberatung auch leisten können bzw. diese angemessen entlohnt werden kann.

Ratsam erscheint, dass Fachberater für Sozialunternehmer selbst FallSupervisionen in Anspruch nehmen bzw. in Intervisionsgruppen nach professionellen Vorgaben kooperieren. Verbände könnten dafür eine Plattform schaffen oder einfordern und dafür Sorge tragen, dass die von ihnen gelisteten Berater nach Qualitätsstandards arbeiten, die auf Beraterportfolios mit der Abgrenzung zu Kompetenzgrenzen beruhen. Bislang bestehen keine Initiativen und mutmaßlich ebenso keine Kompetenz, Ist-Profile mit Anforderungsprofilen abzugleichen. Ebenso könnte nach Vorwissen und Erfahrungslevel der Ratsuchenden unterschieden werden zwischen einer Anfänger- und Fortgeschrittenenberatung. Entsprechende Konzeptionen könnten z. B. in Kooperation mit unabhängigen Lehrstühlen, die sich auf Qualitätssicherung in der Personal- und Organisationsentwicklung spezialisiert haben, ausgearbeitet werden. Da ratsuchende Social Entrepreneure nicht wissen können, inwieweit ein Fachberater einen bestimmten Beratungsbedarf professionell bedienen kann und seine Passgenauigkeit im Schlüssel-Schloss-System gegeben ist, sollte ein entsprechendes digitales Angebot zur Unterstützung geschaffen werden, das dieses Problem reduziert.

Darüber hinaus wurde Supervisionsbedarf für Social Entrepreneure herausgearbeitet, auch als Debriefings zur Nachbereitung schwerwiegender Fehlbelastungen und Krisensituationen bis hin zum Aufarbeiten des sozialunternehmerischen Scheiterns. Gerade bezogen auf Selbst- und Fremdreflexionen, die sich u. a. damit auseinandersetzen können, inwieweit ein harmonisch verschmolzenes Konzept aus „Social + Entrepreneur" machbar oder eben gehemmt erscheint, wird empfohlen, ein entsprechendes Beratungsangebot zu installieren. Zweifelsohne stellt sich hierbei die Frage der Bewältigung einer damit gegebenen finanziellen Mehrbelastung: Zumindest könnten im nächsten Schritt die zumeist universitär angebundenen Unterstützungsorganisationen mit anderen Lehrstühlen kooperieren, die im Beratungswesen entsprechende Kompetenzen mitbringen bzw. mit Ausbildungsinstituten für Supervisoren vernetzt sind. Denn gerade werdende Supervisoren, die noch in ihrer mehrjährigen Aus- bzw. Weiterbildung sind und bei hochwertigen Qualifizierungsprogrammen umfangreiche Praxiserfahrung 
nachweisen müssen, können eventuell für kostenfreie bzw. erschwingliche Supervisionsangebote gewonnen werden, die dennoch professionell gestaltet werden.

Vor allem aber bekunden Befragte einen Beratungsbedarf durch Mentoren mit (branchenspezifischer) Erfahrung im entsprechenden sozialunternehmerischen Gebiet, die systemisch denken und sich auf die Ideenwelt ihrer Mentees einlassen können. Jedoch erscheint es angesichts der Arbeitsüberlastung auf allen Ebenen utopisch, Mentorenschaften hinreichend zu ermöglichen. Umso mehr besteht akuter Handlungsbedarf bei der Qualitätssicherung zu Beratertätigkeiten. Gleichwohl sollten Mentorenprogramme gezielt gefördert und entwickelt werden.

Last, not least bleibt zu hoffen, dass die aus dieser Untersuchung hervorgegangenen Erkenntnisse zum Wirkungsmodell zu Arbeitsgestaltungsbedingungen von Social Entrepreneuren in Deutschland, zu den Typologie-Ansätzen und ebenso die Ausführungen zum prozessorientierten Qualitätsmanagement unter Einbezug des strukturellen Präventionsverständnisses zur Entwicklung von Basisbausteinen für bedarfsorientierte Unterstützungstools in Beratungskontexten herangezogen werden. Denn der hohen Bedeutung von Fehlbelastungen ist ein angemessener Stellenwert zu verleihen.

\section{Literaturverzeichnis}

Ajzen, I. (1991). The Theory of Planned Behavior. In: Organizational Behavior and Human Decision Processes, 50 (2), S. 179-211.

Hockerts, K. (2015). The Social Entrepreneurial Antecedents Scale (SEAS): A Validation Study. In: Social Enterprise Journal, 11 (3), S. 260-280.

Kruse, P., Chipeta, E. M., Surujlal, J. \& Wegge, J. (2018). Measuring Good Intentions - Development and Validation of a Social Entrepreneurial Intention Scale. Unveröffentlichtes Manuskript, eingereicht beim Journal of Business Venturing mit der Manuskript-Nr. JBV_2018_547. Nachgereicht zur Veranstaltung ,What motivates social entrepreneurs? A meta-analysis on predictors of social entrepreneurial intention“ am 17.09.2018 beim 51. DGPs Kongress an der Goethe-Universität in Frankfurt am Main. Session A6: Unternehmertum, Social Entrepreneurial Intention (SEI). Dozent: Philipp Kruse, Fakultät Psychologie an der Technischen Universität Dresden, Professur für Arbeits- und Organisationspsychologie.

Kruse, P., Wach, D., Costa, S. \& Moriano J. A. (2018). Values matter, don't they? Combining Theory of Planned Behavior and Personal Values as Predictors of Social Entrepreneurial Intention. In: Journal of Social Entrepreneurship, 10 (1), S. 55-83.

Lee, M., Battilana, J. \& Wang, T. (2014). Building an Infrastructure for Empirical Research on Social Enterprise: Challenges and opportunities. In: Social Entrepreneurship and Research Methods, 9, S. 241-264.

Leppert, T. (2013). Social Entrepreneurship in Deutschland. Einflussfaktoren auf den Gründungsprozess von Social Entrepreneurs. Hamburg: Kovač. 
Majumdar, S. \& Reji, E. (2020). Introduction: Methodological Issues in Social Entrepreneurship Knowledge and Practice. In: S. Majumdar und E. Reji (Hrsg.), Methodological Issues in Social Entrepreneurship Knowledge and Practice. Singapur (Singapur): Springer Nature, S. 1-11.

Short, J., Moss, T. \& Lumpkin, G. (2009). Research in Social Entrepreneurship: Past Contributions and Future Opportunities. In: Strategic Entrepreneurship Journal, 3 (2), S. 161-194.

Open Access Dieses Kapitel wird unter der Creative Commons Namensnennung 4.0 International Lizenz (http://creativecommons.org/licenses/by/4.0/deed.de) veröffentlicht, welche die Nutzung, Vervielfältigung, Bearbeitung, Verbreitung und Wiedergabe in jeglichem Medium und Format erlaubt, sofern Sie den/die ursprünglichen Autor(en) und die Quelle ordnungsgemäß nennen, einen Link zur Creative Commons Lizenz beifügen und angeben, ob Änderungen vorgenommen wurden.

Die in diesem Kapitel enthaltenen Bilder und sonstiges Drittmaterial unterliegen ebenfalls der genannten Creative Commons Lizenz, sofern sich aus der Abbildungslegende nichts anderes ergibt. Sofern das betreffende Material nicht unter der genannten Creative Commons Lizenz steht und die betreffende Handlung nicht nach gesetzlichen Vorschriften erlaubt ist, ist für die oben aufgeführten Weiterverwendungen des Materials die Einwilligung des jeweiligen Rechteinhabers einzuholen.

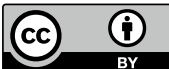

